\title{
Beispiel für eine Herangehensweise zur Vermeidung einer „Rückfallsituation“ bei der Verbesserung der Compliance für die Händehygiene
}

Die Qualitätssicherung für die Durchführung der Händehygiene wurde als ein neuer und wichtiger Aspekt in die aktuellen Empfehlungen der Kommission für Krankenhaushygiene und Infektionsprävention (KRINKO) „Händehygiene in Einrichtungen des Gesundheitswesens" integriert. ${ }^{1}$ Darin wurden „die Maßnahmen zur Verbesserung der Compliance ${ }_{\boldsymbol{1}}$ "zum zentralen Thema gemacht.

Dass die Verbesserung der Händehygiene-Compliance immer noch ein bedeutender Aspekt ist, zeigt auch eine aktuelle Veröffentlichung von von Lengerke et al. "Förderung der hygienischen Händedesinfektion“2. Die Autoren berichten von einer sogenannten „Rückfallsituation“ in Bezug auf die Compliance der hygienischen Händedesinfektion nach einer initialen Compliance-Steigerung auf Intensivstationen (ITS) eines Krankenhauses der Maximalversorgung.

Ziel der oben genannten und nachfolgend zusammengefassten Studie war es, eine Strategie zu entwickeln, um auf zehn ITS und zwei Knochenmark-Transplantationsstationen (KMTS) eines Universitätsklinikums die Compliance der Händehygiene nachhaltig zu verbessern. ${ }^{2}$ Dabei wurden maßgeschneiderte verhaltenspsychologische Interventionen $_{2}$ nach dem Compliance-Modell „Health Action Process Approach“ (HAPA) ${ }^{3-6}$ der „Aktion Saubere Hände“ (ASH) eingesetzt, um eine mögliche Rückfallsituation zu verhindern bzw. ihre Auswirkungen zu minimieren. Zentrale Elemente der maßgeschneiderten verhaltenspsychologischen Interventionen waren die initiale Identifikation der Einflussfaktoren auf das Verhalten der behandelnden Ärzte und des Pflegepersonals und die Durchführung individueller Schulungen und Feedbackgespräche auf Clusterebene.

In einem Cochraine-Review wird berichtet, dass maßgeschneiderte Interventionen häufig bei der Implementierung von Maßnahmen zur Verbesserungen der „Leistungserbringung" von Gesundheitsfachkräften empfohlen werden. ${ }^{7}$ Zum Spektrum möglicher Einflussfaktoren auf das Verhalten der behandelnden Ärzte und des Pflegepersonals werden im o.g. Review ${ }^{7}$ folgende Faktoren gezählt:

- individuelle Faktoren des Gesundheitsfachpersonals (z. B. Wissen, Motivation, Wahrnehmung wahrscheinlicher Vorteile sowie Risiken),

- Patientenfaktoren (z.B. Patientenerwartung, Patientenpräferenz),

- Leistungsanreize,

- Ressourcenverfügbarkeit,

- geeignete Empfehlungen (gut verständlich, die alle wichtigen Aufgabenbereiche abdecken),

- organisatorische Faktoren (z. B. Hilfsmittel, Arbeitsbelastung),

soziale, politische und rechtliche Faktoren. ${ }^{7}$

1 Compliance $=$ Leitlinienkonformität

2 Intervention = geplante und gezielt eingesetzte Maßnahme
Zur Identifikation der Einflussfaktoren auf das Verhalten der Akteure wurden in der PSYGIENE-Studie ${ }^{2}$ im Zeitraum vom 26.11.2012 bis 25.01.2013 Mitarbeiterbefragungen durchgeführt. Der Fragebogen erfasste zentrale psychische Compliance-Determinanten sowie stationsbezogene Ressourcen und Barrieren. Mit den verantwortlichen Stationsärzten und Stationsleitern wurden im Zeitraum vom 19.03.2013 bis 07.05.2013 problemzentrierte Interviews $\mathrm{zu}$ typischen Tagesabläufen/Tätigkeiten geführt. Am Ende stand eine verhaltenspsychologische Analyse der Stärken und Schwächen der einzelnen Akteure sowie der fördernden und hindernden Arbeits- sowie Umweltbedingungen in Anlehnung an die Methode der SWOT-Analyse (SWOT: Strengths, Weaknesses, Opportunities, Threats). ${ }^{8}$

Das Studiendesign sah zwei Studienarme vor: einen Interventionsarm und einen Kontrollarm. In den sechs Stationen des Interventionsarms wurden maßgeschneiderte Verhaltensänderungstechniken (BCT: behavioral change technique $)^{9}$ in Schulungen und Feedbackgesprächen umgesetzt, wohingegen in den sechs Stationen des Kontrollarms Schulungen gemäß ASH durchgeführt wurden. Dabei wurden 15 der 29 BCT eingesetzt, die im Interventionsarm eingesetzt wurden. Auch fanden im Kontrollarm keine Feedbackgespräche statt.

Alle Interventionen fanden vom 20.06.2013 bis 16.12.2013 statt und umfassten alle zum Zeitpunkt der Studie tätigen Ärzte $(n=515)$ und Pflegenden $(n=572)$ in den jeweiligen Stationen (www.aerzteblatt.de/callback/image. asp?id=78837).

Compliance-Raten für die hygienische Händedesinfektion wurden durch Beobachtungen gemäß Goldstandard der WHO von Januar bis Dezember 2014 bzw. von Januar 2015 bis Februar 2016 erfasst. Als primäre Endpunkte dienten die Compliance-Raten von 2014 und 2015. Sekundäre Endpunkte waren die Veränderungen in den Zeiträumen 2013-2014, 2014-2015 und 2013-2015 innerhalb jedes Studienarms.

Die wichtigsten Ergebnisse der Studie unter Betrachtung aller Berufsgruppen waren:

- Die Ausgangs-Compliance im Jahr 2013 war für den Interventions- und Kontrollarm gleich.

- Im Interventionsarm stieg die Compliance in den Jahren 2014 und 2015 jeweils relativ zum Vorjahr an.

- Im Kontrollarm stieg die Compliance im Jahr 2014 an und sank im Jahr 2015 wieder ab.

- Der Compliance-Zuwachs im Zeitraum 2013-2015 sowie die Compliance-Raten im Jahr 2015 waren im Interventionsarm höher als im Kontrollarm. Der Unterschied in den Compliance-Raten zwischen Interventions- und Kontrollarm war für das Jahr 2015 statistisch signifikant. 
Betrachtet man die verschiedenen Berufsgruppen, so gab es bei den Ärzten hinsichtlich des Compliance-Zuwachses im Zeitraum 2013-2015 keine Unterschiede zwischen den beiden Studienarmen. Im Interventionsarm stieg die Compliance in den Jahren 2014 und 2015 an. Im Kontrollarm war im Jahr 2014 ein stärkerer Zuwachs als im Interventionsarm zu messen, und im Jahr 2015 nahm die Compliance im Kontrollarm wieder ab. Der Unterschied in den Compliance-Raten zwischen Interventions- und Kontrollarm war im Jahr 2014 statistisch signifikant, nicht jedoch im Jahr 2015 .

Für die Pflegenden stieg die Compliance im Interventionsarm in den Jahren 2014 und 2015 ebenfalls an. Im Kontrollarm wurde nach einer Compliance-Steigerung im Jahr 2014 - vergleichbar zum Interventionsarm - im Jahr 2015 ein Rückgang - anders als im Interventionsarm - beobachtet. Der Compliance-Zuwachs im Zeitraum 2013-2015 war im Interventionsarm höher.

Zusammenfassend ist die PSYGIENE-Studie eine der wenigen Studien und die erste Studie in Deutschland, die sich des Problems Compliance-Wiederanstieg nach Rückfallsituation angenommen hat. Die maßgeschneiderten Interventionen führten in der Pflege und dadurch auch insgesamt zu nachhaltigeren Compliance-Wiederanstiegen (2-Jahres-Follow-up). Die Autoren der Studie argumentieren, dass die Ergebnisse im Interventionsarm durch Verhaltensänderungstechniken zustande kamen und demzufolge nachhaltiger wirken. Dies sollte durch weitere Studien untermauert werden.

Als Schlüssel für eine langfristige Verbesserung der Compliance im Gesundheitswesen schlussfolgern die Autoren, dass die Händehygiene als fortwährende Kernaufgabe ärztlicher und pflegerischer Tätigkeiten angesehen werden sollte, für deren Erfolg die Kommunikation eine zentrale Rolle spielt. Ferner schlagen die Autoren vor, die Händehygiene als eine Teamaufgabe zu definieren und zu verankern.

\section{Literatur}

1. Kommission für Krankenhaushygiene und Infektionsprävention (KRINKO): Händehygiene in Einrichtungen des Gesundheitswesens - Empfehlung der Kommission für Krankenhaushygiene und Infektionsprävention (KRINKO) beim Robert Koch-Institut (RKI). Bundesgesundheitsbl 2016; 59: 1189-1220; DOI 10.1007/s00103-016-2416-6; http://www.rki.de/DE/ Content/Infekt/Krankenhaushygiene/Kommission/Downloads/Haendehyg_Rili.pdf?_blob=publicationFile

2. von Lengerke T, Lutze B, Krauth C, Lange K, Stahmeyer JT, Chaberny IF: Förderung der hygienischen Händedesinfektion. Clusterrandomisierte kontrollierte Studie PSYGIENE zur Evaluation maßgeschneiderter Interventionen. Deutsches Ärzteblatt 2017; 114 (3): 27-36

3. Aktion Saubere Hände: Händedesinfektion und Compliance: Warum ist eine so einfache Maßnahme so schwierig durchzusetzen? www.aktionsauberehaende.de/ash/module/krankenhaeuser/fortbildungsmaterialien (Zugriff am 09.11.2016)

4. Schwarzer R, Lippke S, Luszynska A: Mechanisms of health behavior change in persons with chronic illness or disability: the Health Action Process Aproach (HAPA). Rehabil Psychol 2011; (56): 161- 170

5. Schwarzer R, Fleig L: Von der Risikowahrnehmung zur Änderung des Gesundheitsverhaltens. Zentralblatt für Arbeitsmedizin, Arbeitsschutz und Ergonomie 2014; 64 (5): 338-341

6. von Lengerke T, Schulz-Stübner S, Chaberny IF, Lutze B: Psychologie der Händehygiene-Compliance: Von der Motivation zum Verhalten. Krankenhaushygiene up2date 2016; 11 (02): 135-150

7. Baker R, Camosso-Stefinovic J, Gillies $C$ et al.: Tailored interventions to address determinants of practice. The Cochrane database of systematic reviews 2015 (4): CD005470

8. van Wijngarden J, Scholten G, van Wijk K: Strategic analysis for healtcare organisations: the suitabillity of SWOT-analysis. Int J Health Plann Manage 2012; (27): 34-39

9. Michie S, Richardson M, Johnston $M$ et al.: The behavior change technique taxonomy $(\mathrm{v} 1)$ of 93 hierarchically clustered techniques: building an international consensus for the reporting of behavior change interventions. Ann Behav Med 2013; 46 (1): 81-95

\footnotetext{
- Dr. Christin Perlitz

Robert Koch-Institut | Abteilung für Infektionskrankheiten | FG 14 - Angewandte Infektions- und Krankenhaushygiene Korrespondenz: PerlitzC@rki.de | aktion-sauberehaende@charite.de

- Vorgeschlagene Zitierweise:

Perlitz C: Verbesserung der Compliance für die Händehygiene: Beispie für eine Herangehensweise zur Vermeidung einer "Rückfallsituation". Epid Bull 2017;18/19: 169-170 | DOI 10.17886/EpiBull-2017-025
} 